\title{
Etude de faisabilité de la mise en place d'un site écotouristique dans le parc national de Boumba-Bek: cas des clairières forestières de Pondo
}

\author{
Mbezele Junior Yannick NGABA ${ }^{1,2}$ et Martin TCHAMBA ${ }^{2 *}$ \\ ${ }^{I}$ Collège de Foresterie, Université d'Agriculture et de Foresterie de Fujian (FAFU), \\ BP 35002, Fuzhou, Fujian, China. \\ ${ }^{2}$ Département de Foresterie, Faculté d'Agronomie et des Sciences Agricoles (FASA), Université de Dschang, \\ B.P. 222 Dschang, Cameroun. \\ *Auteur correspondant ; E-mail: mtchamba@yahoo.fr ; Tel: (+237) 6777705412
}

\section{RESUME}

L'écotourisme, alternative d'impulsion du développement et de financement durable, est une solution viable pour faire face à l'insuffisance de moyens financiers rencontrés par les gestionnaires de parcs nationaux. C'est ainsi que la présente étude s'est donnée pour objectif de savoir si les clairières forestières de Pondo situées dans la partie Sud du Parc National de Boumba-Bek possèdent un potentiel faunique susceptible d'être valorisé. Les méthodes du «scan sampling» et les «recces» ont été utilisées. Il ressort de l'analyse des données qu'un total de 16 espèces, représentées par plusieurs espèces emblématiques, entre autres, Loxodonta africana cyclotis, Gorilla gorilla, Panthera pardus. Les sites sont fréquemment visités en matinée (20,1\%). Loxodonta africana cyclotis a l'abondance relative la plus élevée avec 0,699 indice au kilomètre. Un total de 68 signes d'activités anthropiques a été recensé pour un taux d'IKA moyen de 0,15 indice au $\mathrm{km}$ avec un coefficient de détermination $R^{2}=0,7674$. Le coefficient de corrélation de Spearman $r=-0,8760$ a été obtenu à travers l'équation $\mathrm{y}=-44,144 \mathrm{x}+316,72$. Il ressort de cette étude que les clairières forestières de Pondo sont régulièrement fréquentées et peuvent faire l'objet d'une valorisation écotouristique.

(C) 2019 International Formulae Group. All rights reserved.

Mots clés: Grands et moyens mammifères, clairières forestières, suivi écologique, parc national.

\section{Feasibility study of the implementation eco-tourist site in Boumba-Bek national park: case of Pondo forest clearings}

\begin{abstract}
Ecotourism, an alternative to development stimulus and sustainable financing is a viable solution to address the financial constraints by national park managers. The objective of this study is to determine whether Pondo forest clearings in the southern part of Boumba-Bek national park have wildlife potential that can be harnessed for eco-tourism purposes. The "scan sampling" and "recces" methods were used. Analysis of the data shows that a total of 16 species represented by several emblematic species including Loxodonta africana cyclotis, Gorilla gorilla, Panthera pardus. Sites were frequently visited in the morning (20,1\%) and Loxodonta
\end{abstract}


africana cyclotis has the highest relative abundance with 0,699 index per kilometer. A total of 68 signs of anthropogenic activity were identified for an average IKA rate of 0,15 index per kilometer with a coefficient of determination $\mathrm{R}^{2}=0,7674$. The correlation coefficient of Spearman $r=-0,8760$ was obtained from equation $y=-44,144 x+316,72$. This study shows that Pondo forest clearings are regularly used and can be used for ecotourism purposes.

(C) 2019 International Formulae Group. All rights reserved.

Keywords: Large and medium mammals, forest clearings, ecological monitoring, national park.

\section{INTRODUCTION}

Les forêts du Bassin du Congo, considérées comme le deuxième poumon de la planète, sont parmi les écosystèmes les plus riches de la terre (FAO, 2011). Elles abritent plusieurs habitats et une forte diversité animale soit 400 espèces de mammifères, 1086 espèces d'oiseaux, 216 espèces d'amphibiens et 280 espèces de reptiles (Tchatchou, et al., 2015). Le Parc National de Boumba-Bek (PNBB), appartenant à ce massif forestier représente l'un des derniers vestiges de forêt vierge et abrite une grande variété des espèces animales parmi lesquelles on retrouve plusieurs espèces emblématiques telles que les éléphants, les gorilles et les chimpanzés. De façon spécifique, il abrite 37 espèces de grands mammifères dont 11 espèces de primates, 12 espèces d'ongulés et 04 espèces de carnivores (MINFOF, 2011 ; Nzooh et al., 2016). Malheureusement, ce périmètre de conservation de la biodiversité fait face à plusieurs menaces (braconnage, chasse sportive professionnelle, extension des voies d'accès dans les unités forestières d'aménagement (UFA) périphérique au parc, prolifération d'armes à feu dans la sousrégion, empiétement, insuffisance de financement, exploitation forestière non durable et mal organisée entraînant la fragmentation des habitats de la faune, perturbation des couloirs de migrations des éléphants ...). Elles pourraient en l'absence de réponses appropriées, compromettre sa richesse et empêcher au PNBB d'atteindre ses objectifs de gestion. En effet, des études ont relevé que les prélèvements de chasse effectués sur certaines espèces animales sont largement supérieurs à l'optimum de prélèvement naturel possible (Fouda, 2009).
De plus, à cause du contexte de la pauvreté, les braconniers s'attachent généralement aux services de la population allochtone (Baka et Bantou) pour leur connaissance du milieu (Billand, 2005 ; MINFOF, 2011). Bien que certaines organisations internationales engagées dans la conservation à l'instar du Fond Mondial pour la Nature (WWF) jouent un rôle important comme partenaire d'appui, le budget alloué au service de conservation pour la mise en place et en œuvre du dispositif de gestion est insuffisant en ce sens que cette somme ne permet pas de couvrir les charges récurrentes nécessaires à l'atteinte des objectifs de conservation visés (MINFOF, 2011). Dès lors, il est primordial de trouver une solution fiable susceptible de faire face simultanément aux enjeux de réduction de la pauvreté et de conservation qui permettra de garantir la pérennité de ce patrimoine tout en soutenant le bien-être des populations locales. La valorisation économique de la ressource faunique à travers l'écotourisme apparaît ainsi comme un des moyens viables pouvant concourir à l'atteinte de cet idéal car il est perçu comme un secteur de l'économie en pleine croissance sur le marché international (Tchindjang et al., 2006). En effet, un modèle de développement au service de la conservation peut être ainsi considéré comme un outil de lutte contre la pauvreté et une solution alternative de financement durable dont peuvent profiter plusieurs acteurs: la communauté riveraine, le service de conservation du PNBB et le gouvernement Camerounais. Par ailleurs, le PNBB est composé d'un complexe de clairières forestières marécageuses localement appelées «Baïs» par les peuples Baka qui sont des ouvertures en forêt sur des sols marécageux 
ou affleurements rocheux, hydromorphes ou non hydromorphes généralement riches en sels minéraux. Ces milieux abritent à l'intérieur des forêts tropicales des habitats distincts dont la végétation diffère considérablement de celle des habitats voisins (Temgoua et al., 2018). Elles abritent parfois des salines et la végétation est généralement dominée par les herbacées (Eno, 2001; Ngenyi, 2002). Ces spécifications de son environnement font d'elles une zone de «hotspot» car elles représentent pour plusieurs espèces animales (éléphants de forêt, gorilles de plaine, buffles, bongos, sitatungas, hylochères, potamochères, etc.) un lieu d'alimentation, de récréation, de villégiature, de reproduction, de refuge et d'échanges inter et intraspécifiques. Ainsi, la facilité qu'offrent ces sites pour les observations des espèces animales peut être valorisée pour le développement du tourisme de vision. Des études ont déjà été menées dans certaines clairières du PNBB telles Batouka, Djewa, Likolo I et Likolo II mais aucune n'a été faite dans les clairières se situant dans la zone de Pondo (Ngaba, 2015). C'est dans ce contexte que cette étude s'est donnée pour but de valoriser ce milieu afin qu'il remplisse au mieux ses fonctions. La valorisation de ce milieu passe nécessairement par la caractérisation des sites, la composition spécifique des grands et moyens mammifères présentes dans cette zone et les types de menaces qui y sont exercées. La finalité de ces travaux est de savoir si le potentiel faunique de Pondo peut garantir un espace viable et soutenable pour le développement de l'écotourisme.

\section{MATERIEL ET METHODES}

\section{Localisation de la zone d'étude}

L'étude a été réalisée dans le complexe des clairières forestières de Pondo situé entre $2^{\circ} 45^{\prime}$ et $2^{\circ} 48^{\prime}$ latitude Nord et entre $14^{\circ} 98^{\prime}$ et $15^{\circ} 01^{\prime}$ longitude Est dans la partie Sud du PNBB à près de $1500 \mathrm{~m}$ de la rivière Bek, affluent de la grande rivière Dja (Figure 1) qui la sépare de la zone d'intérêt cynégétique (ZIC) n³8. Le PNBB fait partie de l'Unité Technique opérationnelle localisé dans la partie Sud-Est au Cameroun. Le climat est de type équatorial humide, fortement marqué par la continentalité avec 04 saisons et les précipitations s'étalent tout au long de l'année avec deux pics en avril et en octobre (Harrison et Agland, 1987 ; Ekobo, 1995). La température annuelle moyenne est de $24{ }^{\circ} \mathrm{C}$ et l'humidité relative de l'air varie de 60 à $90 \%$.

Le système hydrographique coule vers le Sud jusqu'à la rivière Dja puis Ngoko et deux affluents du fleuve Congo. Les sols de la zone appartiennent au sous-ordre des sols ferrallitiques typiques définis par une séparation du fer et de l'oxyde d'alumine, sont de type argileux avec une fine couche d'humus superficiels contenant peu de matière organique. Ils sont acides et pauvres en azote et bases échangeables (Noupa et al., 2008).

\section{Description et présentation générale des clairières de Pondo}

L'étude menée dans le complexe de Pondo était composée de 04 principaux sites à savoir : 03 clairières forestières sans saline (clairière $n^{\circ} 1, n^{\circ} 2$ et $n^{\circ} 3$ ) et 01 clairière forestière avec saline. Les clairières de la zone d'étude sont à sol hydromorphe, périodiquement inondées et possèdent une végétation prédominée par un tapis herbacé. Dans la plupart des clairières, la végétation est composite, constituée de graminées, d'astéracées, de fougères, de cypéracées et de quelques marantacées caractéristiques des marécages (Figure 2A). Cependant, la végétation des lisières des clairières est constituée par des arbres tels que Mellettia mannii, Maytenus sp, Diospyros sp. Anonidium mannii, Uapaca paludosa, Cola rostrata, etc.

La clairière forestière avec saline est constituée d'un sol non hydromorphe, dominée par une végétation herbacée et des cailloux avec un cours d'eau qui traverse une partie du site (Figure 2B).

\section{Méthodologie}

L'étude a été menée du 18 mai au 03 septembre 2014 durant 20 jours par mois soit 
un total de 60 jours d'observation et l'intervalle de temps entre les descentes étaient de 10 jours. Pour la collecte des données, deux méthodes ont été combinées. La méthode du scan sampling (Turkalo et Fay, 1995 ) et les recces ou marches de reconnaissance (White et Edwards, 2000). La méthode du scan sampling a été utilisée afin de recueillir les informations sur la grande faune fréquentant les clairières de la zone de Pondo tandis que celle des recces a permis de relever les signes d'activité animales et anthropiques; d'évaluer l'intensité et l'impact de la pression anthropique sur la dynamique des espèces de grands et moyens mammifères. La méthode de recces consistait à se déplacer dans l'aire d'étude sur des chemins de moindre résistance (pistes d'animaux, pistes d'hommes, sous-bois clairs, lignes de crêtes, etc.) dans une direction prédéterminée suivant un cap donné (Walsh et White, 1999). Chaque

$$
\text { FOD }=\frac{\text { Nombre d'espèces observées }}{\text { Nombre total de jours de mission }}
$$

observation était collectée sur des fiches et géoréférencées. Afin d'avoir une meilleure vision sur la dynamique des populations animales, les ratios d'âge ont été appréciés et calculés. Dans le cadre de ce travail, trois classes d'âges ont été considérées: adulte, sub-dulte et juvénile en se basant sur le dimorphisme sexuel. Au cas où un individu n'a pas pu être classé dans l'une des trois catégories, il était systématiquement classé comme "Indéterminé". Au niveau des sites, les observations directes des animaux étaient faites à l'aide de jumelles entre $07 \mathrm{~h}$ et $17 \mathrm{~h} 30$ à partir d'un mirador situé à $8 \mathrm{~m}$ du sol.

\section{Analyses statistiques}

Le taux de rencontre ou IKA moyen (indice kilométrique d'abondance)

Les indices kilométriques d'abondance (Bousquet, 1996) nous ont permis d'estimer l'abondance relative des populations animales et des activités anthropiques de la zone d'étude. Elles ont été calculées avec la formule suivante :

$$
\text { IKA }=\frac{\text { Nombre de contact avec une espèce }}{\text { Distance totale parcourue }}
$$

\section{L'indice de diversité de Simpson}

Afin de mesurer la richesse spécifique de la zone, l'indice de diversité de Simpson a été calculé. Cet indice est donné par la formule suivante :

$D=1-\sum\left[\frac{\left(n_{k}\left(n_{k}-1\right)\right)}{(n(n-1))}\right]$

Où $\mathrm{n}=$ nombre d'animaux observés, $\mathrm{n}_{\mathrm{k}}=$ nombre d'animaux de l'espèce $\mathrm{k}$.

Le taux de présence journalier $\left(\mathrm{TP}_{k}\right)$, le taux de fréquentation $\left(\mathrm{TF}_{k}\right)$ et la fréquence d'observation directe (FOD).

Ils ont été calculés calculés avec les formules suivantes :

$$
\begin{gathered}
\mathrm{TF}_{\mathrm{k}}=\frac{\text { Nombre total d'individus d'une espèce } \mathrm{k}}{\text { Nombre total d'individus observés }} \times 100 \\
\mathrm{TP}_{\mathrm{k}}=\frac{\text { Nombre de jours de présence d'une espèces } \mathrm{k}}{\text { Nombre de jours d'observation }} \times 100
\end{gathered}
$$

Le logiciel Excel 2016 a été utilisé pour l'encodage et le tri des données, les logiciels SPSS v20.0 et Statistica v.12 pour l'analyse des données. La carte de localisation des sites a été faite via le logiciel ArcGIS 10.3. L'analyse de variance à un facteur a été utilisée pour évaluer s'il y a une différence significative des populations animales entre les sites pilotes. 


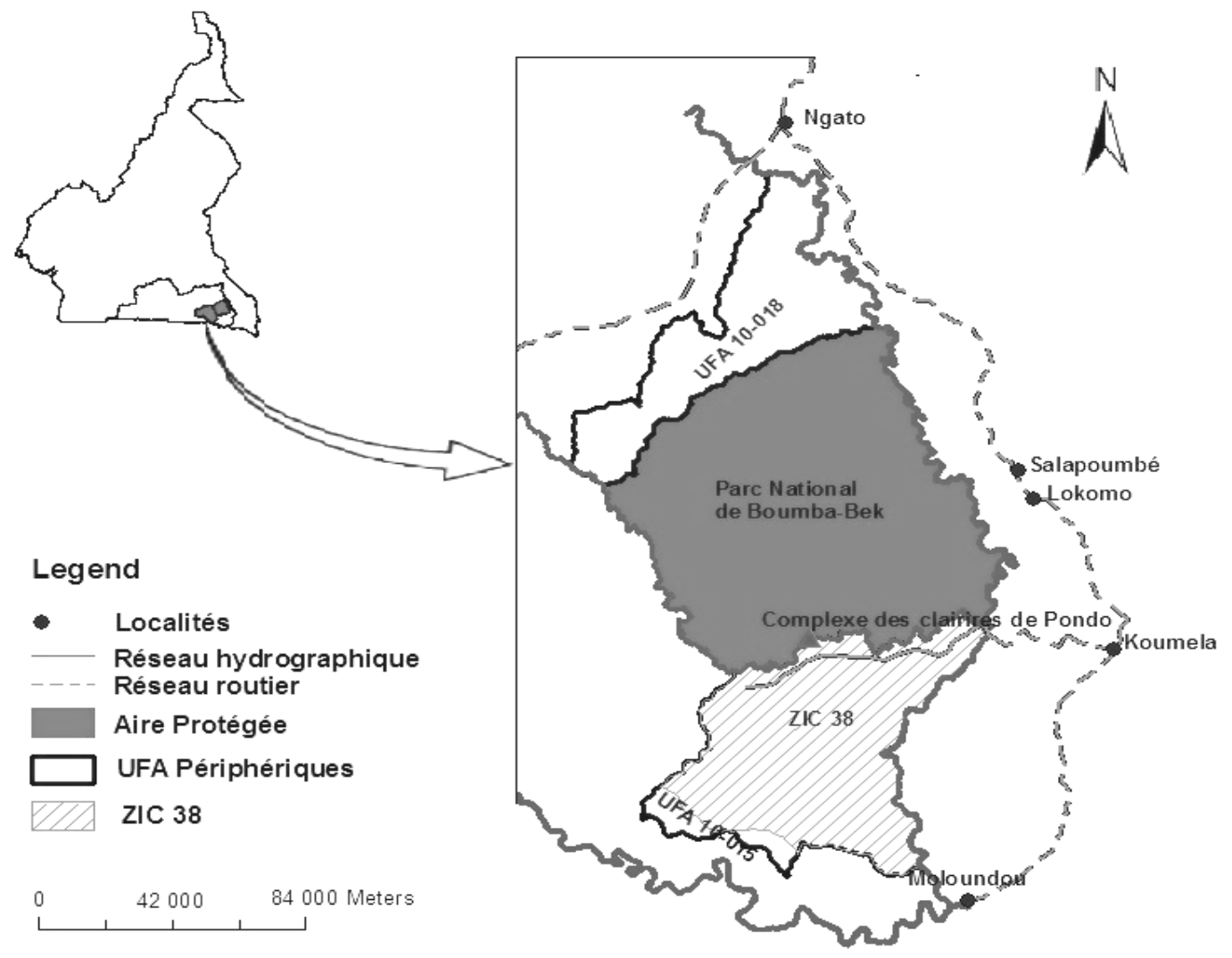

Figure 1 : Carte du site de l'étude.
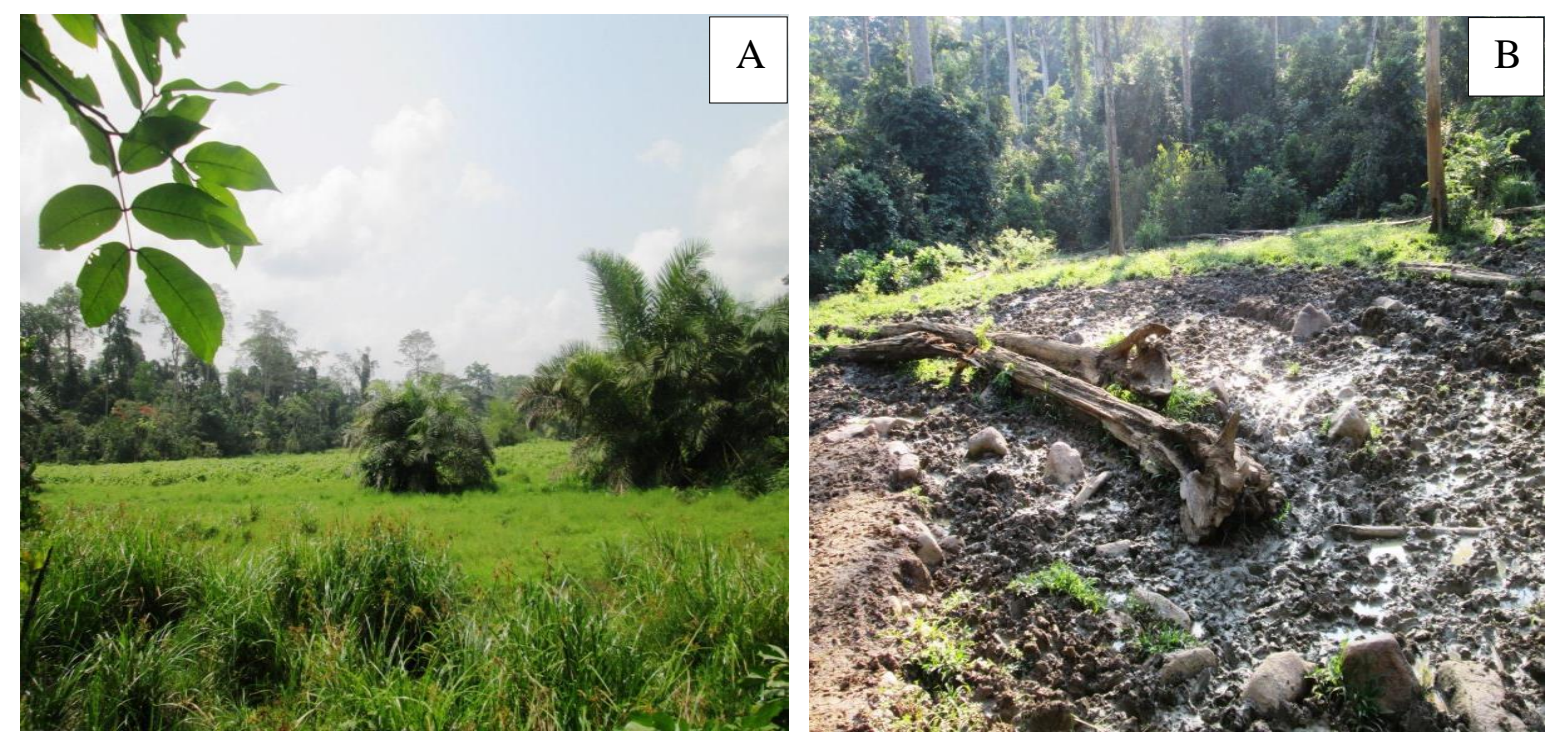

Figure 2 : Présentation des sites d'étude. A: Clairière sans saline $n^{\circ} 2$ (à gauche), B: Clairière forestière avec saline $n^{\circ} 3$ (à droite). 


\section{RESULTATS}

\section{Les clairières forestières de Pondo}

\section{Description générale des sites d'observation dans les clairières de Pondo}

L'étude nous a permis d'identifier et de caractériser 04 sites dans la zone, la distance qui les sépare variait entre 3 à $7 \mathrm{~km}$. Il ressort des analyses que 551 animaux ont été le plus observés dans la clairière forestière avec saline soit $(47,89 \%)$, suivi de la clairière $\mathrm{n}^{\circ} 1$ $(24,18 \%)$, la clairière $n^{\circ} 3(18,18 \%)$ et la clairière $\mathrm{n}^{\circ} 2(9,75 \%)$ (Table 1). L'utilisation du test $F$ au seuil de probabilité de 5\% montre qu'il existe une différence significative entre les valeurs moyennes des signes d'activités animales observées dans les différents sites (ANOVA un facteur, $F_{3}=11,575, P=0,003$ ). La plus petite différence significative des observations directes utilisée montre qu'il y a une différence significative entre la clairière forestière avec saline et les clairières forestières sans saline $\mathrm{n}^{\circ} 1, \mathrm{n}^{\circ} 2$ et $\mathrm{n}^{\circ} 3(P=$ $0,001, P=0,001$ et $P=0,002)$. Par ailleurs, il n'existe pas une différence significative entre la clairière forestière sans saline $\left(\mathrm{n}^{\circ} 1, \mathrm{n}^{\circ} 2\right.$ et $\mathrm{n}^{\circ} 3$ ). L'indice de diversité de Simpson a été estimé à 0,88 .

\section{La richesse spécifique de la zone d'étude}

Le complexe de Pondo est constitué d'une grande richesse spécifique, un total de 16 espèces a été recensé, soit une représentativité de $43 \%$ d'espèces de grands et moyens mammifères parmi ceux répertoriées par Nzooh et al. (2016) dans le PNBB. Elles sont réparties dans 04 ordres (Artiodactyles, Proboscidiens, Carnivora et Primates), 08 familles (Bovidae, Traguilidae, Suidae, Éléphantidae, Félidae, Cercopithecidae, Colobidae et Pongidae) et 03 classes de protection existantes au Cameroun (Table 2).

\section{Fréquence de visite et temps de visite des}

\section{animaux dans les sites et la fréquence}

\section{d'observation directe (FOD)}

Les observations directes ont été portées sur 11 espèces tandis que la présence de 5 autres espèces a été signalé par des signes indirectes (empreintes, crottes, vocalisation...). Un total de 151 observations directes a été effectué lors de cette étude soit $14,71 \%$ sur le nombre d'observations totales. Il ressort de l'analyse des données que la durée de visite des animaux dans les sites variait entre 01 à 30 minutes selon le moment de la journée, l'espèce, le climat et l'activité menée. Les gorilles ont été les animaux ayant mis le plus de temps dans le site précisément dans la clairière forestière avec saline, avec 30 minutes suivi des buffles (17 minutes) et des magistrats (09 minutes). Il a été observé, par ailleurs, que les sites sont plus visités dans la matinée (69\%) que dans l'après-midi (31\%) plus précisément entre $10 \mathrm{~h}$ et $11 \mathrm{~h}(20,1 \%)$.

L'analyse des données fait ressortir 03 classes d'espèces animales en fonction de leur fréquence d'observation (Figure 3). Les espèces facilement observables : le gorille (Gorilla gorilla), magistrat (Colobus guereza), le buffle nain de forêt (Syncerus caffer nanus), le céphalophe bleu (Cephalophus monticola), l'hocheur (Cercopithecus nictitans) et le moustac (Cercopithecus cephus). Les espèces moyennement observables : Le céphalophe à dos jaune (Cephlophus silvicultor), le céphalophe à bande dorsale noire (Cephalophus dorsalis); le chevrotain aquatique (Hyemoschus aquaticus), le cercocèbe agile (Cercocebus agilis), le sitatunga (Tragelaphus spekei) et les espèces difficilement observables : l'éléphant de forêt (Loxodonta africana cyclotis), le bongo (Boocercus euryceros), le potamochère (Potamochoerus porcus), le chimpanzé (Pan troglodytes) et la panthère (Panthera pardus).

\section{Statut des grands et moyens mammifères Les indices kilométriques d'abondance}

Les observations indirectes des indices lors des marches de reconnaissance ont permis de dénombrer 916 (85,29\% des observations totales) signes d'activité sur une distance de 7,3 indices au $\mathrm{km}$ parcouru pendant 60 jours, soit une distance totale de $438 \mathrm{~km}$. Il ressort de là que l'éléphant a l'abondance relative la plus élevée avec 0,699, suivi du buffle nain 
$(0,596)$ et du gorille signes $(0,242)$ indices au kilomètre.

\section{Comportement et structure d'âge des animaux : cas spécifique des gorilles}

Les animaux observés venaient pour diverses raisons: s'alimenter, s'abreuver, se reposer, ou pour se distraire parfois en groupe ou seul (Figure 4) et la distance de fuite des animaux rencontrés variait de $17 \pm 8 \mathrm{~m}$ selon l'espèce rencontrée et le couvert végétal. Les gorilles ont été l'espèce ayant passé le plus de temps dans la clairière forestière avec saline. Deux groupes ont pu être clairement distingués notamment par leur taille. Il a été observé de multiples simulations de combat et de jeux généralement entre les jeunes, ceux-ci émettaient des cris et se frappaient la poitrine comme moyen d'intimidation ou de domination de leur adversaire. Les femelles, quant à elles étaient sur leur garde et restaient près des plus petits tandis que le mâle dominant généralement au dos argenté restait toujours en retrait du groupe et se positionnait le plus souvent à la lisière du site.

Cependant, plusieurs juvéniles et mâles solitaires ont également été aperçus ce qui est un bon indicateur sur l'état de santé de la dynamique de leur population (Figure 5). Pour les heures de fréquentation des gorilles dans les sites, il ressort des analyses que la probabilité d'observer les gorilles dans la zone est plus élevée en matinée $(67,4 \%)$ que dans l'après-midi (14\%) précisément entre $9 \mathrm{~h}$ et 10 $\mathrm{h}$ pour une durée d'observation de $7 \pm 3$ minutes (Figure 6).

\section{Taux de fréquentation d'observation des animaux dans les sites}

Les données ont été réparties en trois groupes selon la fréquence d'observation des espèces. On a ainsi : les espèces régulières (13-20\%), les espèces non régulières (7-13\%) et les espèces rares (0-13\%) (Figure 7). Les espèces régulières sont au nombre de 04 parmi lesquelles le gorille (Gorilla gorilla) avec $18,5 \%$ suivi du magistrat (Colobus guereza) avec $17,2 \%$, le buffle nain de forêt (Syncerus caffer nanus) avec $13,9 \%$, et le céphalophe bleu (Cephalophus monticola) avec 11,9\%. Les espèces non régulières sont au nombre de 06, parmi lesquelles le moustac (Cercopithecus cephus) avec 8,6\%, l'hocheur (Cercopithecus nictitans) avec 7,9\%, le sitatunga (Tragelaphus spekei) avec 7,9\%, le chevrotain aquatique (Hyemoschus aquaticus), le céphalophe à dos jaune (Cephlophus silvicultor) et le céphalophe à bande dorsale noire (Cephalophus dorsalis).

Les espèces rares sont au nombre de 06 parmi lesquelles figurent l'éléphant de forêt (Loxodonta africana cyclotis), le bongo (Boocercus euryceros), le potamochère (Potamochoerus porcus), le Cercocèbe agile (Cercocebus agilis), le chimpanzé (Pan troglodytes) et la panthère (Panthera pardus). Par ailleurs, l'éléphant de forêt (Loxodonta africana cyclotis) et le buffle nain de forêt (Syncerus caffer nanus) ont le taux d'observation des indices de présence le plus élevé avec respectivement $41,9 \%$ et $32,9 \%$.

\section{Etat des lieux des activités anthropiques}

Plusieurs signes d'activités anthropiques ont été répertoriés. Il s'agit notamment des empreintes de pas d'individus des coupes de machettes, des sachets de plastique et des coups de feu qui ont été entendus. Un total de 68 indices a été recensé pour un taux d'IKA moyen de 0,15 indices au kilomètre. La Figure 8 nous présente le coefficient de détermination $\left(\mathrm{R}^{2}=0,767\right)$. Il ressort de cette analyse que les activités anthropiques ont une forte et négative influence sur la dynamique de la population animale.

Le coefficient de corrélation de Spearman $(r=-0,8760)$ quant à lui nous montre que les activités anthropiques ont un impact négatif sur la dynamique de population de grands et moyens mammifères dans la zone de Pondo à travers l'équation $\mathrm{y}=-44,144 \mathrm{x}+$ 316,72 . 
Table 1: Abondance relative et signes de présence animale par sites et selon les types d'observation de Juin à Août 2014.

\begin{tabular}{lccc}
\hline Site & Total des indices & Fréquence $\mathbf{( \% )}$ & IKA \\
\hline Saline & 511 & & \\
Clairière $n^{\circ} 1$ & 258 & 47,89 & 365 \\
${\text { Clairière } \mathrm{n}^{\circ} 2}^{\circ}$ & 104 & 24,18 & 151,8 \\
Clairière $\mathrm{n}^{\circ} 3$ & 194 & 9,75 & 28,9 \\
Total & 1067 & 18,18 & 323,3 \\
\hline
\end{tabular}

Table 2: Composition spécifique de la faune sauvage recensée du site d'étude.

\begin{tabular}{|c|c|c|c|c|}
\hline Ordres & Familles & Noms scientifiques & Noms communs & $\begin{array}{l}\text { Statut de } \\
\text { protection }\end{array}$ \\
\hline \multirow{8}{*}{ Artiodactyles } & \multirow{6}{*}{ Bovidae } & Cephalophus silvicultor & Céphalophe à dos jaune & $\mathrm{A}$ \\
\hline & & Cephalophus dorsalis & $\begin{array}{l}\text { Céphalophe à bande } \\
\text { dorsale noire }\end{array}$ & B \\
\hline & & Bocerus eurycerus & Bongo & B \\
\hline & & Syncerus caffer nanus & Buffle nain & B \\
\hline & & Tragelaphus spekii & Sitatunga & B \\
\hline & & Cephalophus monticola & Céphalophe bleu & $\mathrm{C}$ \\
\hline & Traguilidae & Hyemoschus aquaticus & Chevrotain aquatique & A \\
\hline & Suidae & Potamochoerus porcus & Potamochère & B \\
\hline Proboscidiens & Éléphantidae & Loxodonta africana cyclotis & Éléphant de forêt & A \\
\hline \multirow[t]{2}{*}{ Carnivora } & Félidae & Panthera pardus & Panthère & A \\
\hline & & Cercocebus agilis & Cercocèbe agile & A \\
\hline \multirow{5}{*}{ Primates } & Cercopithecidae & Cercopithecus cephus & Moustac & $\mathrm{C}$ \\
\hline & & Cercopithecus nictitans & Hocheur & $\mathrm{C}$ \\
\hline & Colobidae & Colobus guereza & Magistrat & A \\
\hline & \multirow[t]{2}{*}{ Pongidae } & Gorilla gorilla & Gorille & A \\
\hline & & Pan troglodytes & Chimpanzé & A \\
\hline
\end{tabular}




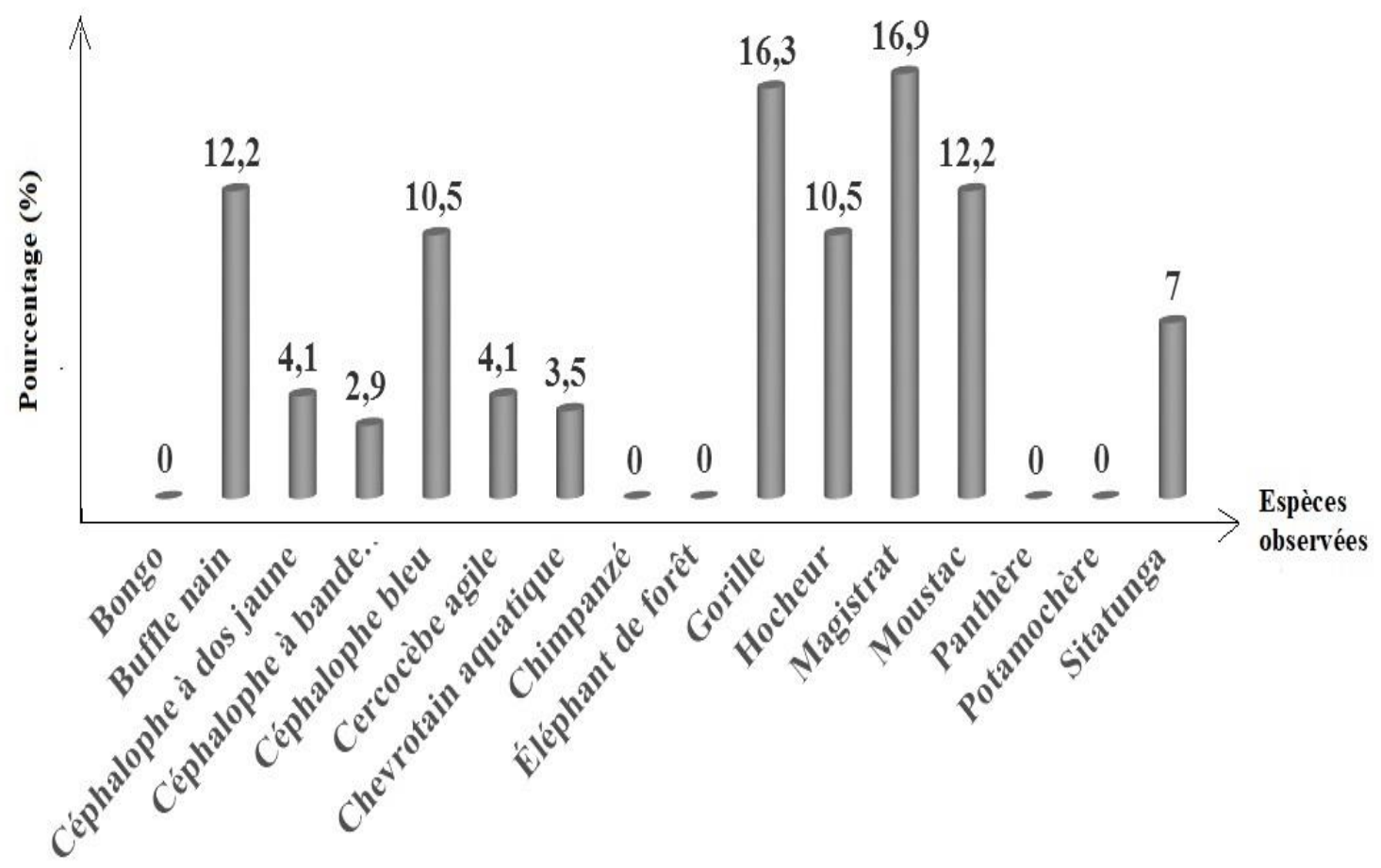

Figure 3 : Fréquence d'observation directe des animaux dans le complexe de Pondo.
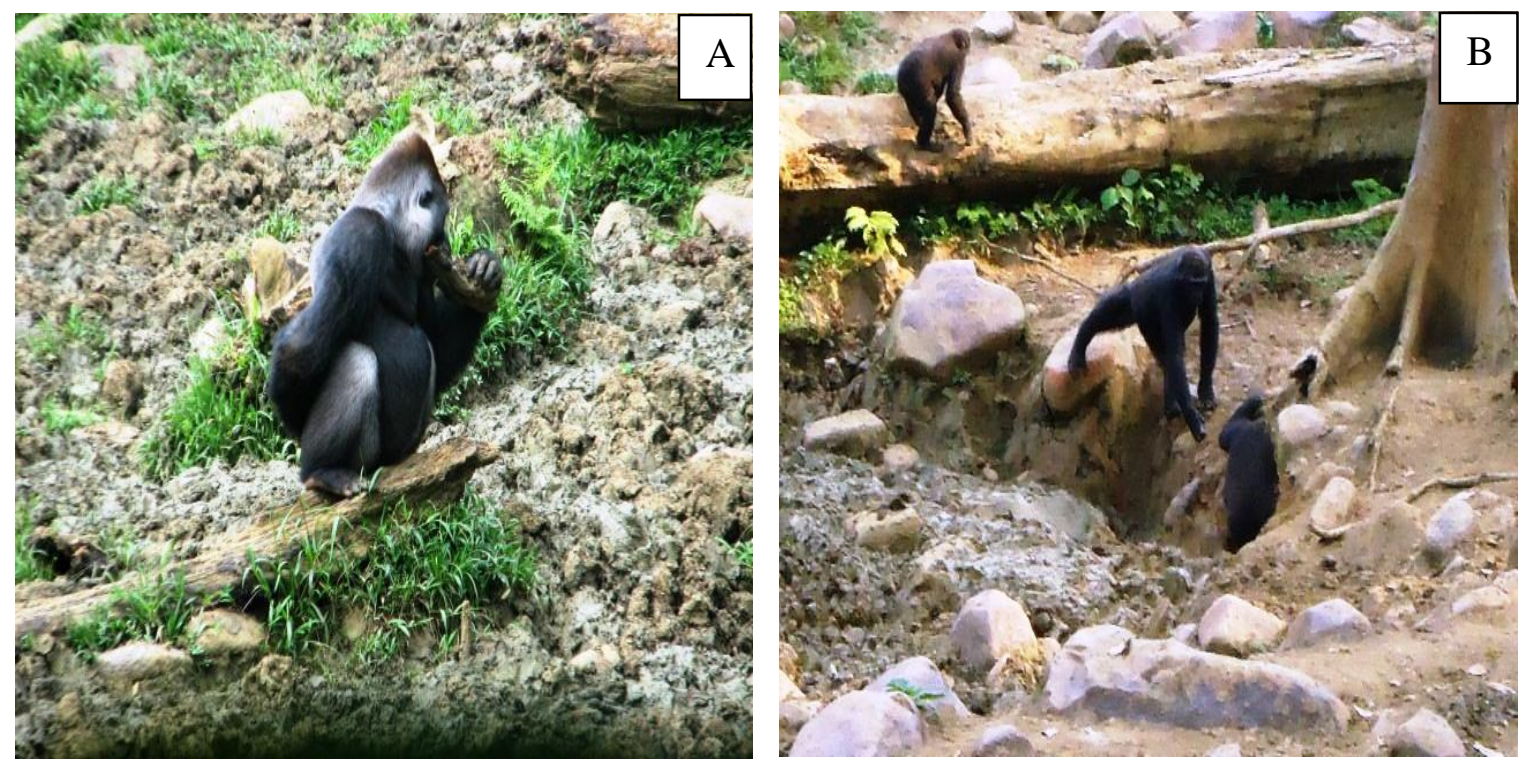

Figure 4 : Activités des gorilles dans la clairière avec saline. A : Mâle adulte solitaire. B : Gorilles en train de s'alimenter. 


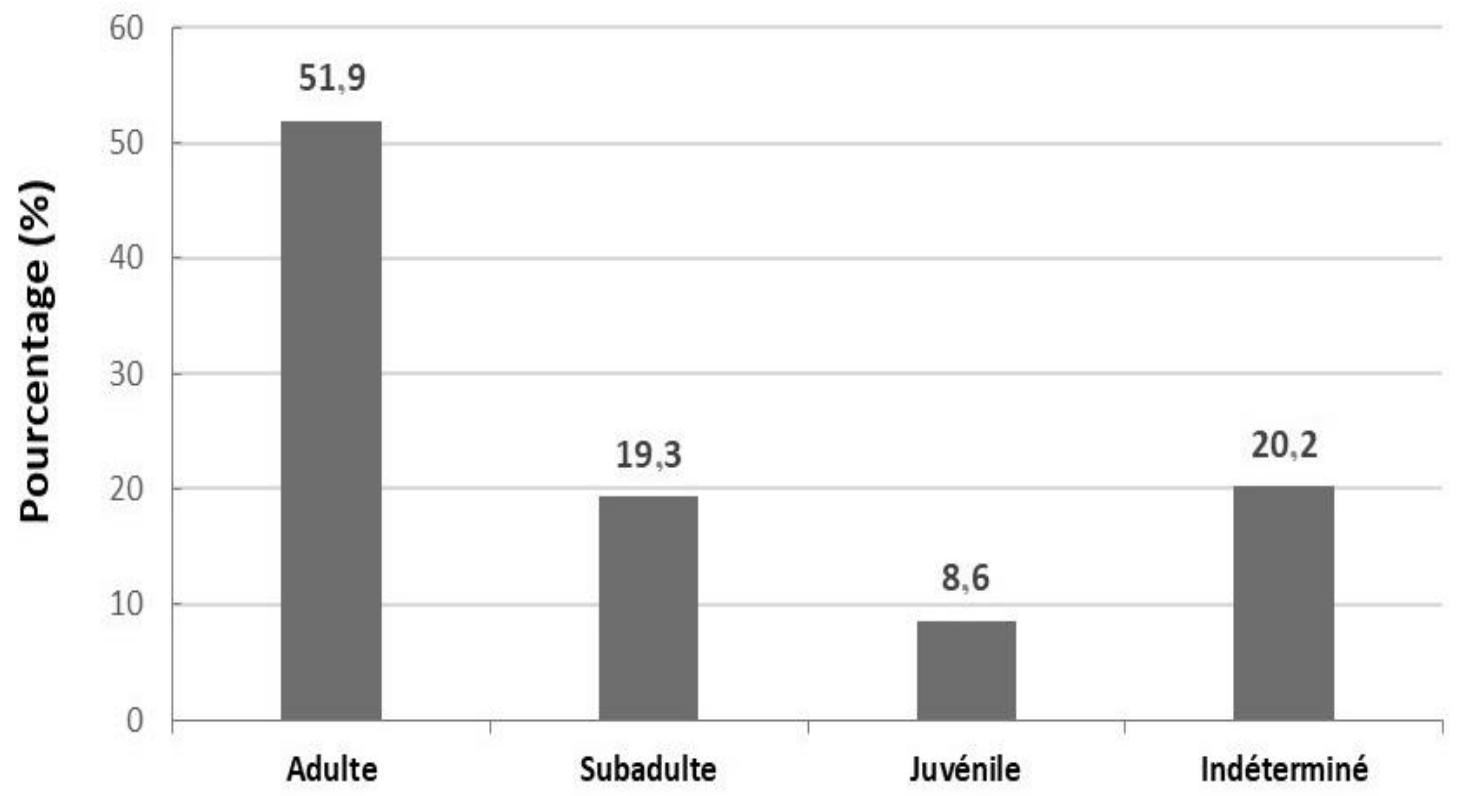

Age

Figure 5 : Structure d'âge des gorilles dans la zone d'étude.

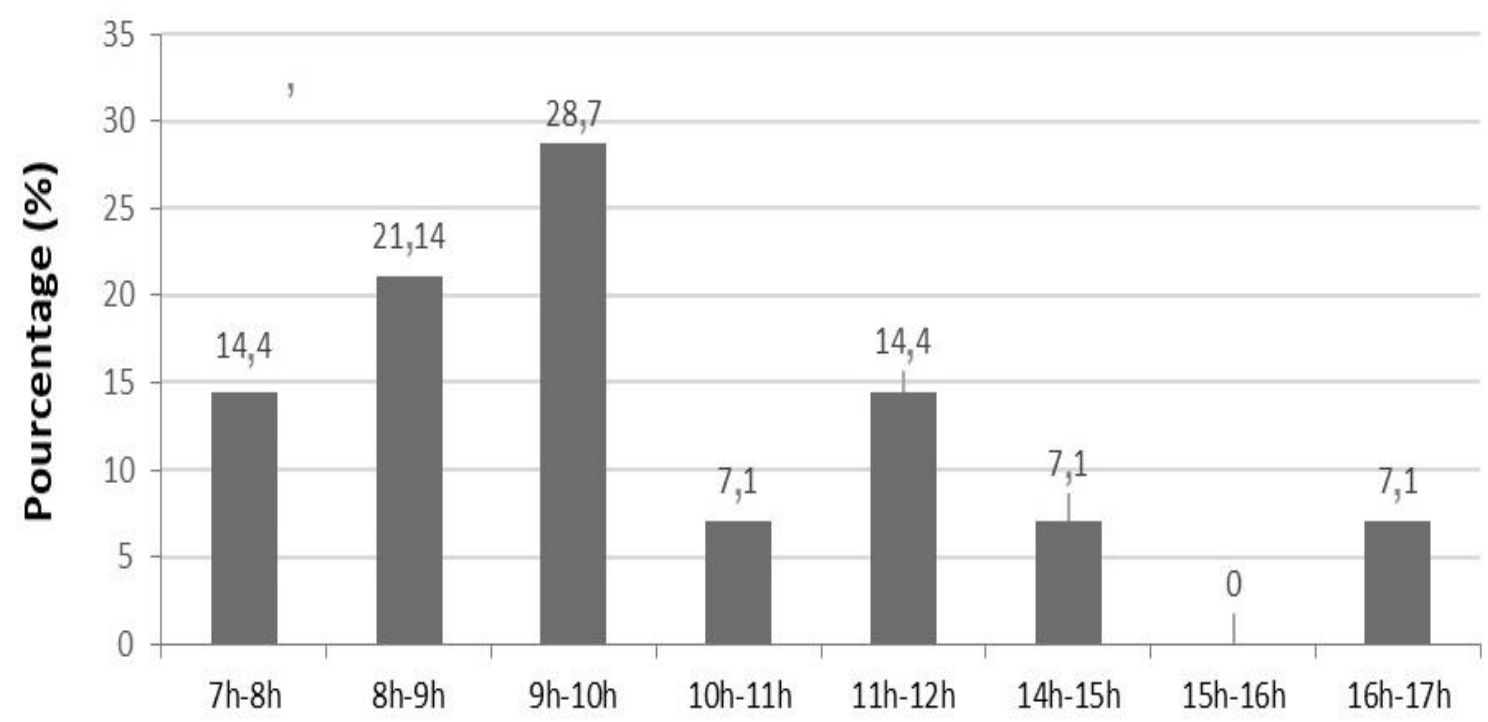

\section{Heures}

Figure 6: Heures de fréquentation des gorilles dans la zone d'étude. 


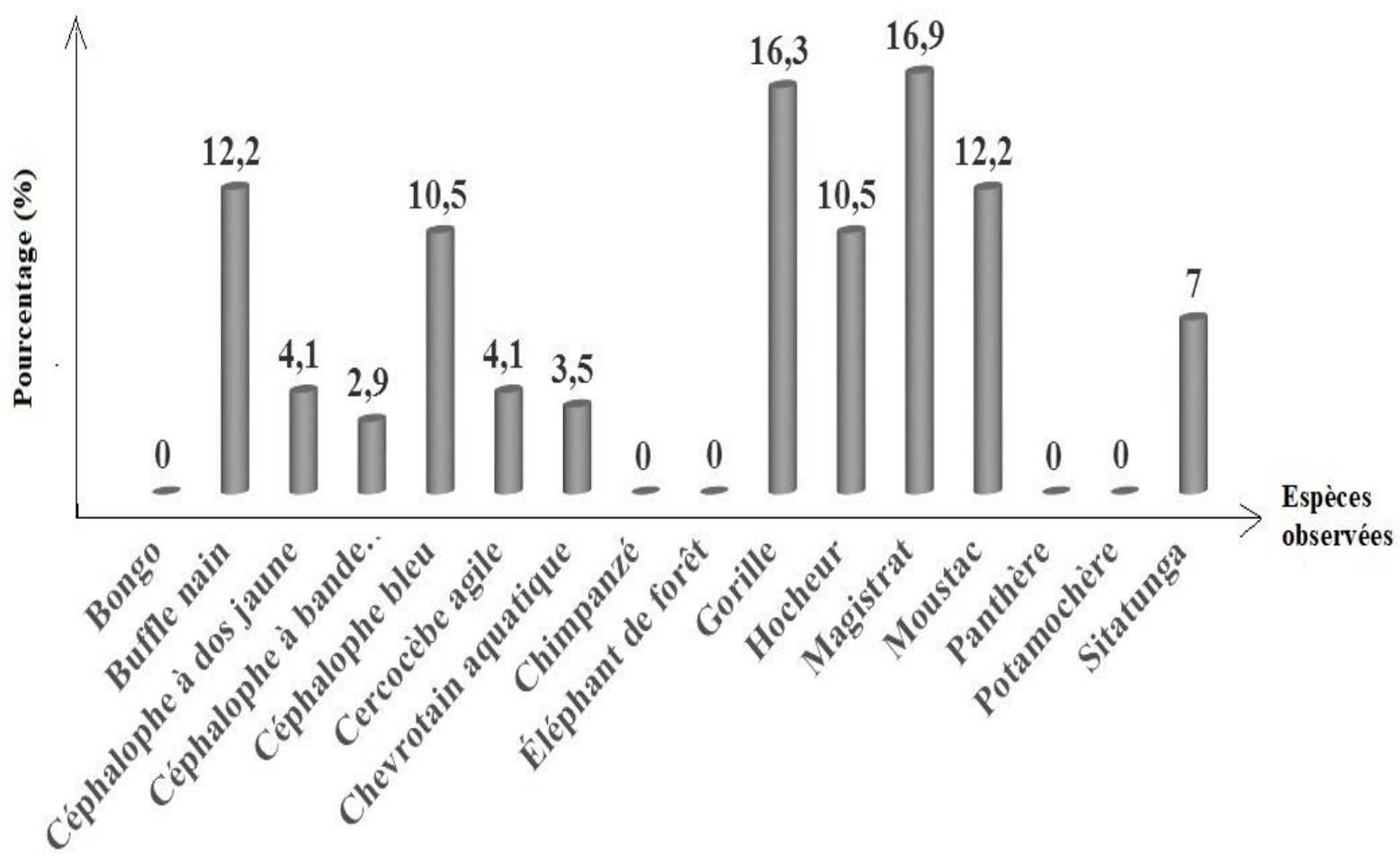

Figure 7 : Fréquence d'observations des signes de présence par espèces dans le complexe de Pondo.

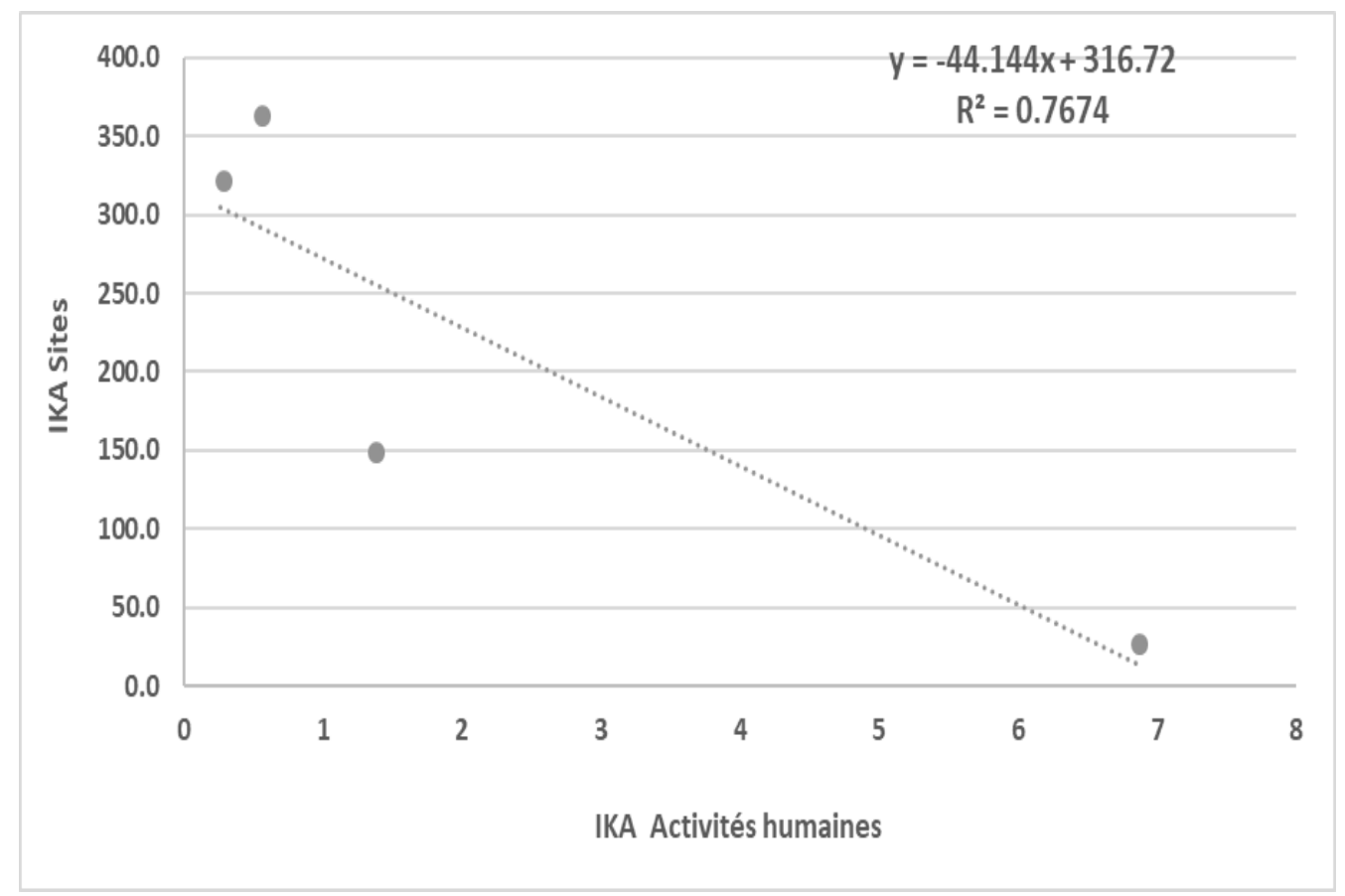

Figure 8: Droite de régression entre les activités anthropiques et la dynamique de population animale. 


\section{DISCUSSION}

\section{Richesse spécifique et fréquence de visite des animaux dans la zone d'étude}

$\mathrm{Au}$ total, 16 espèces de grands et moyens mammifères sur les 37 recensées lors d'une l'étude mené par Nzooh et al. (2016) ont été identifiées dans la zone. Cette différence peut s'expliquer à travers l'étendue de l'aire d'étude restreint à la zone de Pondo. En effet, l'étude menée par Nzooh et al. (2016) a été faite dans l'ensemble du PNBB or la présente étude est restreinte qu'à sa partie Sud. Ce qui limite et réduit la diversité d'habitats étudiés et par conséquent la possibilité d'observer certaines espèces animales. Par ailleurs, la clairière forestière avec saline a le taux d'animaux observés le plus élevé avec 14 espèces soit $87,5 \%$ ce qui indique qu'il offre le meilleur cadre pour l'épanouissement des animaux présentant ainsi un intérêt particulier pour certains animaux tel que l'éléphant (Angoni et al., 2018). Deux raisons majeures peuvent être soulevées : la nature du site qui est caractérisé par un sol hydromorphe au centre du site contrairement aux clairières forestières sans saline gorgées d'eau (Figure $2 \mathrm{~A})$ avec des zones pouvant aller à plus de 1 $\mathrm{m}$ de profondeur et la présence d'une végétation herbacée (Figure $2 \mathrm{~B}$ ) très prisée par les herbivores tels que les buffles. De plus, on note la présence de salines riches en sels minéraux notamment sodium, calcium et magnésium des eaux boueuses généralement prisées par les éléphants qui constituent un caractère attractif pour les animaux de la zone.

Il a été observé, en outre, que les animaux visitent beaucoup plus les sites en journée entre 8 h et 12 h. En effet, les clairières ont un paysage spécifique marqué entre autres par l'absence de canopée qui pourtant sert de bouclier contre l'intensité des rayons lumineux. C'est sans doute pour cette raison que les animaux préfèrent visiter les sites en matinée car le climat est favorable. Contrairement aux clairières de Likolo où des observations directes d'éléphants ont été faites (Effila et al., 2014), l'éléphants et le bongo n'ont pas été aperçus lors de l'étude bien que des indices frais de leur passage ont été observés chaque jour dans la zone d'étude. Cette attitude de déplacement nocturne dans la zone est un indicateur de la pression qui est exercée sur eux par les braconniers et un indicateur du caractère adaptatif des animaux qui ont changé leur mode de déplacement afin de faire face à cette menace.

\section{Statut des grands et moyens mammifères}

Le taux élevé d'IKA moyen des éléphants, de bongo et des buffles peut s'expliquer par la physionomie des espèces car ceux-ci se déplacent généralement en groupe, ce qui donne une consistance des empreintes au sol et les indices sont facilement observables dus à leur poids. Toutefois, il convient de relever que l'abondance relativement faible de certains mammifères tels que les hocheurs, le cercocèbe agile ou la panthère est susceptible d'être sous-estimée car leurs signes d'activités sont difficilement perceptibles à cause de leur mode de déplacement furtif ou sur les arbres. Il en est de même de l'abondance relative des espèces inféodes (sitatunga, chevrotin aquatique) qui se déplacent généralement dans des zones gorgées d'eau. Il ressort par ailleurs de l'analyse des données que la densité des traces augmente au fur et à mesure que l'on se rapproche des clairières forestières, traduisant l'influence de celles-ci sur la répartition de la population animale et créant une zone de forte densité animale ou «zone de Hotspot». Cette observation a également été faite sur d'autres clairières forestières du PNBB. En effet, d'après Noupa et al. (2008) la présence des clairières forestières provoquerait une concentration animale quatre à six fois plus grande en son centre dans un rayon de 5 kilomètres dans les clairières de Batouka et Likolo. Le gorille est considéré dans la zone du Sud-Est comme un tabou par les populations locales (Tsakem et al., 2015). Cette croyance constitue ainsi une conversation naturelle pour cette espèce.

\section{Etat des lieux des activités anthropiques}

La distance de fuite élevée des animaux indique une présence anthropique effective qui 
a été confirmée à travers l'observation de multiples indices (empreintes de pas, coupes de machette, paquet de cigarette, feux de brousse...). Un total de 68 indices a été recensé pour un IKA moyen de 0,15 indices au kilomètre. Ces résultats sont différents de ceux trouvés par Bene et Nzooh (2005) avec 0,8 indice au $\mathrm{km}$. Toutefois, les coups de feu enregistrés peuvent avoir été effectués par les braconniers ou par les acteurs des Safaris qui pratiquent leur activité à près de $5 \mathrm{~km}$ de la périphérie Sud du parc national précisément dans la zone d'intérêt cynégétique $n^{\circ} 38$. Les clairières de Pondo restent jusqu'à l'heure épargnées par le grand braconnage car aucune carcasse d'animaux n'a été trouvé malgré le fort taux de fréquentation des éléphants et autres grands mammifères, ce qui n'est pas le cas à Ikwa et Moussoubou ou plusieurs carcasses d'éléphants ont été trouvées (Belinga, 2014). Cette différence peut s'expliquer par l'accessibilité à la zone d'étude. En effet, situé à près de $70 \mathrm{~km}$, la rivière Bek joue un «effet bouclier» à deux niveaux contre toute tentative de pénétration à la zone Sud du parc car elle sépare le parc naturel à sa zone périphérie et la zone périphérie aux villages riverains. De plus, le comité de vigilance mis sur pied par les autorités effectue une fouille systématique de tout individu lors de la traversée du village pour la zone périphérique. Toutefois, les campements observés dans la zone périphérique constituent une situation préoccupante pour les gestionnaires du PNBB. En effet, les occupants de ses campements allument des feux de brousse de façon récurrente et anarchique qui peuvent avoir un impact néfaste sur le paysage forestier et par conséquent sur l'écosystème (Gueguim et al., 2018) surtout en saison sèche. Ces feux sont généralement allumés pour la cuisson des aliments, la lutte contre le froid et pour éloigner les animaux. Par ailleurs, des activités de collecte de produits forestiers non-ligneux (PFNL) ont également été observées dans les périphéries du parc. Malheureusement, peu de données sont disponibles sur les plantes utilisées par les populations locales sur dans le traitement traditionnel et la distribution spatiale. D'autres études ont rencontré des difficultés similaires (Bayaga et al., 2017 ; Lamy et al., 2018). Il est dès lors crucial de faire une étude sur l'importance de la médecine traditionnelle pour les populations locales et la distribution spatiale des espèces botaniques utilisées.

\section{Pondo, site écotouristique ?}

Cette étude nous révèle que, Pondo peut effectivement être valorisé par l'écotourisme précisément à travers le tourisme de vision. Le tourisme de vision est une meilleure forme de valorisation des espèces sauvages est un outil important de développement pour les communautés locales (Macfie \& Williamson, 2010). Toutefois, cette possibilité ne peut être effective, efficace que si certaines mesures sont prises et mise en place. Ces mesures permettront de garantir que les populations locales bénéficieront des retombées économiques générées par la conservation à l'instar des populations riveraines au parc nationale de Lobéké (Tsakem et al., 2015). Notamment, la sécurisation de la zone qui permettra de maintenir la dynamique de fréquentation des animaux tout en garantissant un espace viable pour les visiteurs. Par ailleurs, l'ignorance des sanctions encourues prescrites par la loi et le faible niveau de vie des populations riveraines au PNBB sont des facteurs qui facilitent leur mise en scène dans le processus de braconnage. Ainsi, on ne pourrait atteindre ces objectifs sans passer par la sensibilisation de ceux-ci sur les sanctions qu'ils encourent lors de telles pratiques. L'éveil des consciences de leur rôle clé dans la lutte contre le braconnage et leur engagement pour les générations futures qui doivent bénéficier dans l'avenir des mêmes avantages qu'ils jouissent aujourd'hui est indispensable. $\mathrm{La}$ matérialisation de leur implication peut être effective si cette activité à un impact perceptible dans leur quotidien, notamment l'amélioration de leurs conditions de vie et s'ils sont placés comme acteurs principaux dans le processus de gestion. 
Etant donné que cet espace ne peut être géré comme une entité isolée, car il existe une interconnectivité entre Pondo, le reste du PNBB et sa zone périphérique il est primordial d'avoir une meilleure connaissance $\mathrm{du}$ paysage du PNBB en général et de la zone de Pondo en particulier. D'où la nécessité d'effectuer des études complémentaires et régulières sur la taille de la population, la dynamique de déplacement des animaux et ethnobotanique. Ces études permettront de savoir, entre autres, les couloirs de migration des animaux, les périodes de visite des sites dans le temps et dans l'espace suivant les saisons, le suivi des menaces qui pèsent sur elles et les stratégies à adopter pour y faire face de façon efficace. Toutefois, au vu du taux élevé de la population de gorilles dans la zone et de leur comportement, le processus d'habituation pourrait se présenter comme un outil de développement dans le secteur écotouristique.

\section{Conclusion}

Il ressort de cette étude que les clairières forestières de Pondo abritent une forte richesse spécifique composée de 16 espèces réparties dans toutes les classes de protection au Cameroun dont plusieurs espèces emblématiques entre autres l'éléphant de forêt, le gorille de plaine et la panthère. Les sites sont régulièrement visités par les espèces mais de façon inégale car la clairière forestière avec saline attire la plus grande variété animale, est le site le plus visité et où la probabilité d'observer les animaux est une évidence. La matinée, précisément entre $8 \mathrm{~h}$ et $11 \mathrm{~h}$, est la période de la journée la plus indiquée pour effectuer des observations. De plus, la durée d'observations des animaux peut aller jusqu'à 30 minutes selon l'espèce et le site d'observation. Néanmoins, certains animaux sont difficilement observables comme le bongo ou l'éléphant de forêt qui pourtant sont des espèces qui visitent régulièrement les sites, ce qui indique un déplacement nocturne au regard des indices fraîchement retrouvés. $\mathrm{Ce}$ caractère noctambule induit une pression anthropique sur elle qui a été confirmée par l'observation de traces humaines. Garantir la rentabilité économique de ce milieu pourrait avoir un « effet papillon » en ce sens qu'il permettra au PNBB de faire face à un double enjeu lié au changement de formes d'utilisation des terres (exploitation minière, forestière, agriculture, et les pêches) et celui de la conservation.

\section{CONFLIT D'INTERETS}

Les auteurs déclarent n'avoir aucun conflit d'intérêts.

\section{CONTRIBUTIONS DES AUTEURS}

MJYN et MT ont préparé le concept de l'étude et planifier des descentes de terrain pour la collecte des données. MJYN a analysé les données et préparé les résultats. MJYN et MT ont révisé le document final.

\section{REMERCIEMENTS}

Nous exprimons notre reconnaissance aux responsables du Département de Foresterie, Faculté d'Agronomie et des Sciences Agricoles (FASA) de l'Université de Dschang, aux services de conservation du PNBB et PNK. Les éditeurs anonymes qui ont apportés une contribution significative à l'amélioration de ce travail.

\section{REFERENCES}

Angoni H, Ongolo RS, Melingui JBN, Ngo Mpeck ML. 2018. Composition floristique, structure et menaces de la végétation de la ligne côtière de la Réserve de Faune de Douala-Edéa. Int. J. Biol. Chem. Sci., 12(2): 915-926. DOI: https://dx.doi.org/10.4314/ijbcs.v12i2.2.

Bayaga HN, Guedje NM, Biye EH. 2017. Approche ethnobotanique et ethnopharmacologique des plantes utilisées dans le traitement traditionnel de l'ulcère de Buruli à Akonolinga (Cameroun). Int . J. Biol. Chem. Sci., 11(4): 1523-1541. DOI:

https://dx.doi.org/10.4314/ijbcs.v11i4.1.

Bene Bene CL, Nzooh ZL. 2005. Suivi écologique dans le Parc National de Boumba Bek et sa zone périphérique: 
données de base sur la dynamique des populations de grands et moyens mammifères et des activités anthropiques. Rapport WWF Cameroun report/CCPO.

Belinga JP. 2014. Monitoring au parc national de Nki (clairières Ikwa et Moussoubou). Rapport WWF Cameroun report/JengiTRIDOM.

Billand A. 2005. Etude sur le plan pratique d'aménagement des forêts naturelles de production tropicales africaines: application au cas de l'Afrique Centrale. Aspects faunistiques 3 (Volet 3), Paris, ATIBT, 99p.

http://www.atibt.com/pdf/plan_amenage ment_atibt_Volet_III_francais.pdf

Bousquet B. 2002. Evaluation du Projet Assistance à la Gestion des Ressources Naturelles : composante réhabilitation / réintroduction de la faune sauvage. Ministère chargé des Eaux et Forêts, GTZ projet Assistance à la gestion des ressources naturelles, $63 \mathrm{p}$.

Effila B. 2014. Suivi des grands et moyens Mammifères dans les clairières de la Zone est du parc national de BoumbaBek. Rapport WWF Cameroun report/Jengi-TRIDOM.

Ekobo A. 1995. Conservation of the African forest elephant (Loxodonta Africana cyclotis) in Lobeke, Southeast Cameroon. $\mathrm{PhD}$ thesis, University of Kent.

https://www.memoireonline.com/04/17/9

832/m_Inventaire-de-la-faune-sauvage-

sur-les-transects-permanents-en-

peripherie-nord-est-et-dans-leparc81.html.

Eno Nku M. 2001. Frequency of large mammals visits to forest clearing: a case study of ecotourism potentials in CampoMa'an National Park, southern Cameroun. Master of Science Thesis.

FAO. 2011. Situation des forêts du monde 2011. Rapport FAO, Rome, Italie, 193 p. http://www.fao.org/3/a-i2000f.pdf

Fouda E. 2009. Rapports semestriels des activités du Parc National de Boumba-
Bek et sa zone périphérique. Rapport WWF Cameroun report/Jengi-TRIDOM, Yokadouma.

Gueguim CD, Tchamba MN, Fotso CR. 2018. Dynamique spatio-temporelle des feux de brousse dans le Parc National du Mbam et Djerem (Cameroun). International Journal of Biological and Chemical Sciences, 12(2): 728-748. DOI: https://dx.doi.org/10.4314/ijbcs.v12i2.10.

Harrisson M, Agland P. 1987. A draft proposal for the designation of three new national forest parks. Dja river Films, LTD.

Lamy LGM, Ibrahima A, Ndjonka D, Mapongmetsem PM. 2018. Etude ethnobotanique des sous-variétés de Syzygium guineense (Will.) DC. var. macrocarpum (Engl.) F. White dans les Hautes Savanes Guinéennes (Adamaoua, Cameroun). International Journal of Biological and Chemical Sciences, 12(4): $1636-1649 . \quad$ DOI: https://dx.doi.org/10.4314/ijbcs.v12i4.11.

Macfie EJ, Williamson EA. 2010. Lignes directrices pour de meilleures pratiques en matière de tourisme de vision des grands singes. Groupe de spécialistes des primates de la CSE/UICN, Gland, Suisse.

MINFOF. 2011. Plan d'aménagement du parc national de Boumba Bek et de sa zone périphérique. Période d'exécution : 2012-2016. MINFOF.

Ngaba MJY. 2015. Suivi écologique de la dynamique des grands et moyens mammifères dans les clairières du parc national de Boumba-Bek : Cas du complexe de Pondo. Thèse de Master. DOI : 10.13140/RG.2.2.36383.61600.

Ngenyi A. 2002. Commerce du perroquet gris au Cameroun. Psitta Scene, 14(51) : 2-3.

Noupa P, Nkongmeneck BA. 2008. Influence des clairières forestières sur la répartition spatiale des grands mammifères dans la forêt dense du Bassin du Congo : cas du Parc National de Boumba-Bek (Sud-est Cameroun). International Journal of 
Biological and Chemical Sciences, 2(2): 185-195. DOI: 10.4314/ijbcs.v2i2.39734.

Nzooh ZL, N'goran, Etoga G, Belinga JP. 2016. Les populations de grand et moyens mamifères dans le segment Cameroun du paysage TRIDOM (forets de Ngoyla MINTOM, PN Boumba-Bek, PN Nki et leurs périphériques). Rapport WWF Cameroun.

Tchatchou B, Chia EL, Kankeu RS, Terán ASP, Tiani AM, Sonwa DJ, Kengoum F, Locatelli B, Mekou YB, Munoh A, Kenfack C. 2015. Changement climatique dans le Bassin du Congo: informations et connaissances échangées entre les acteurs. Document de Travail 185 du Center for International Forestry Research

(CIFOR). https://www.cifor.org/library/5622/.

Tchindjang M, Abossolo SA, Armathée J, Menga VF. 2006. Les difficultés de développement de l'écotourisme dans les aires protégées du Cameroun. Boletim Goiano de Geografia, 26(2): 12-47. https://dialnet.unirioja.es/descarga/articul o/4785646.pdf

Temgoua LF, Solefack MCS. 2018. Caractérisation de la végétation des clairières sur sol hydromorphe du Parc National de Lobéké, Est-Cameroun. International Journal of Biological and Chemical Sciences, 12(3): 1364-1380.
DOI:

https://dx.doi.org/10.4314/ijbcs.v12i3.24.

Turkalo A, Fay JM. 1995. Studying elephants by direct observations: preliminary results from the Dzanga clearing, Central African Republic. Pachyderm, 20: 45-54. https://cmsdata.iucn.org/downloads/pach y20.pdf\#page $=48$.

Tsakem SC, Tchamba M, Weladji RB. 2015. Les gorilles du Parc National de Lobéké (Cameroun): interactions avec les populations locales et implications pour la conservation. International Journal of Biological and Chemical Sciences, 9(1): 270-280.

DOI: http://dx.doi.org/10.4314/ijbcs.v9i1.24.

Walsh PD, White L. 1999. What it will take to monitor forest elephant populations. Conservation Biology, 13(5): 1194-1202. DOI: $\quad 10.1046 / j .1523-$ 1739.1999.98148.x.

White L, Edwards A. 2000. Conservation en Forêt Pluviale Africaine : Méthodes de Recherche. Wildlife Conservation Society : New York. 